\title{
Infection of bat and human intestinal organoids by SARS-CoV-2
}

\author{
Jie Zhou ${ }^{1,2,6 凶}$, Cun Li ${ }^{2,6}$, Xiaojuan Liü2,6, Man Chun Chiu ${ }^{2}{ }^{2}$, Xiaoyu Zhao ${ }^{2}{ }^{2}$, Dong Wang ${ }^{2}$, \\ Yuxuan $\mathrm{Wei}^{2}$, Andrew Lee ${ }^{1}{ }^{2}$, Anna Jinxia Zhang, ${ }^{1,2}$, Hin Chu1,2, Jian-Piao Cai ${ }^{2}$, Cyril Chik-Yan Yip ${ }^{2}$, \\ Ivy Hau-Yee Chan ${ }^{3}$, Kenneth Kak-Yuen Wong ${ }^{3}{ }^{3}$, Owen Tak-Yin Tsang ${ }^{4}$, Kwok-Hung Chan ${ }^{2}{ }^{2}$, \\ Jasper Fuk-Woo Chan ${ }^{1,2,5}$, Kelvin Kai-Wang To ${ }^{1,2,5}$, Honglin Chen ${ }^{1,2}$ and Kwok Yung Yuen ${ }^{1,2,5}$
}

\begin{abstract}
A novel coronavirus-severe acute respiratory syndrome coronavirus 2 (SARS-CoV-2)-emerged in humans in Wuhan, China, in December 2019 and has since disseminated globally ${ }^{1,2}$. As of April 16, 2020, the confirmed case count of coronavirus disease 2019 (COVID-19) had surpassed 2 million. Based on full-genome sequence analysis, SARS-CoV-2 shows high homology to SARS-related coronaviruses identified in horseshoe bats ${ }^{1,2}$. Here we show the establishment and characterization of expandable intestinal organoids derived from horseshoe bats of the Rhinolophus sinicus species that can recapitulate bat intestinal epithelium. These bat enteroids are fully susceptible to SARS-CoV-2 infection and sustain robust viral replication. Development of gastrointestinal symptoms in some patients with COVID-19 and detection of viral RNA in fecal specimens suggest that SARS-CoV-2 might cause enteric, in addition to respiratory, infection ${ }^{3,4}$. Here we demonstrate active replication of SARS-CoV-2 in human intestinal organoids and isolation of infectious virus from the stool specimen of a patient with diarrheal COVID-19. Collectively, we established the first expandable organoid culture system of bat intestinal epithelium and present evidence that SARS-CoV-2 can infect bat intestinal cells. The robust SARS-CoV-2 replication in human intestinal organoids suggests that the human intestinal tract might be a transmission route of SARS-CoV-2.

A novel coronavirus, SARS-CoV-2, has caused a global pandemic of COVID-19 since its emergence in December 2019 $19^{1,2,5}$. Common symptoms of COVID-19 include fever, cough, shortness of breath, myalgia and fatigue ${ }^{6}$. Some patients have suffered gastrointestinal symptoms such as nausea, vomiting and diarrhea. Viral RNA was detected in patients' respiratory and stool specimens $^{6-8}$. Full-genome sequence analysis revealed that SARS-CoV-2 clusters with severe respiratory syndrome-related coronaviruses (SARSr-CoVs) found in bats, showing 96\% identity to the bat coronavirus BatCoV RaTG13 and $88 \%$ identity to two other bat SARSr-CoVs (Bat-SL-CoVZC45 and Bat-SL-CoVZXC21) ${ }^{1,8,9}$, suggesting that SARS-CoV-2 might originate in bats.

Bats are natural reservoirs for a variety of viruses, especially RNA viruses, that cause serious disease in humans and animals ${ }^{10,11}$. Extensive search for the progenitor of SARS-CoV, which caused an epidemic in 2003 , led to the discovery of large numbers of related
\end{abstract}

coronaviruses from fecal specimens or anal swabs of Chinese horseshoe bats ${ }^{12,13}$. These discoveries, however, typically depend on the detection of viral genome or genome fragments using molecular approaches. Except for three strains isolated using interferon (IFN)-deficient Vero E6 or Vero cells ${ }^{13,14}$ and one strain of genetically synthesized virus ${ }^{15}$, to date, most identified bat SARSr-CoVs have not been successfully isolated and cultivated. Despite many reports of bat primary cell cultures and immortalized bat cell lines ${ }^{16}$, as well as the commercially available bat cell lines in ATCC, isolation of SARSr-CoV using a bat cell line has never been documented.

The horseshoe bat species Rhinolophus spp, especially Rhinolophus sinicus ( $R$. sinicus), has been proposed to be the natural host of SARS-CoV ${ }^{12,14,17}$. However, no direct evidence exists of SARSr-CoV infection in these bats, which might be owing to the nature of acute and self-limiting infection, seasonal fluctuation of virus growth in these bats ${ }^{18}$ and the difficulty in accessing these animals in the wild. Thus, the absence of a readily accessible in vitro model that can faithfully represent native bat cells is a major obstacle to isolate and study bat viruses ${ }^{16}$, including bat SARSr-CoVs, which are predicted to possess high zoonotic potential ${ }^{15}$.

The past decade has witnessed a major breakthrough in the generation of organoids ${ }^{19}$. The adult stem cell (ASC)-based organoid culture system has been established for most human organs, including the generation of human intestinal organoids and lung organoids ${ }^{20,21}$. While the undifferentiated intestinal organoids are expandable for more than 1 year, upon induction the differentiated intestinal organoids faithfully simulate the multicellular composition and functional complexity of human intestinal epithelium. Because the human gastrointestinal tract is one of the most common routes of microbial invasion, human intestinal organoids have become popular in vitro tools for modeling enteric infections ${ }^{21-23}$. In this study, we attempted to establish an ASC-based organoid culture of bat intestinal epithelium. We propose that bat organoids could be instrumental for experimentally probing the potential origin of SARS-CoV-2. In addition, we investigated the possibility of enteric infection by SARS-CoV-2 in human intestinal organoids.

Considering the high identity of SARS-CoV-2 and bat SARSr-CoVs, and the discovery of SARSr-CoVs in fecal specimens of horseshoe bats ${ }^{12,14}$, we attempted to establish intestinal organoid culture using crypts isolated from the intestines of $R$. sinicus bats.

'State Key Laboratory of Emerging Infectious Diseases, The University of Hong Kong, Hong Kong, China. ${ }^{2}$ Department of Microbiology, Li Ka Shing Faculty of Medicine, The University of Hong Kong, Pokfulam, Hong Kong, China. ${ }^{3}$ Department of Surgery, Li Ka Shing Faculty of Medicine, The University of Hong Kong, Hong Kong, China. ${ }^{4}$ Department of Medicine and Geriatrics, Princess Margaret Hospital, Hong Kong, China. ${ }^{5}$ Carol Yu Centre for Infection,

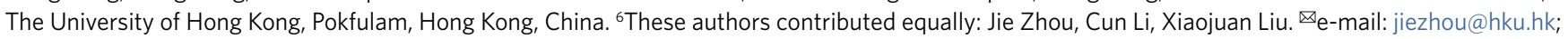
kyyuen@hku.hk 

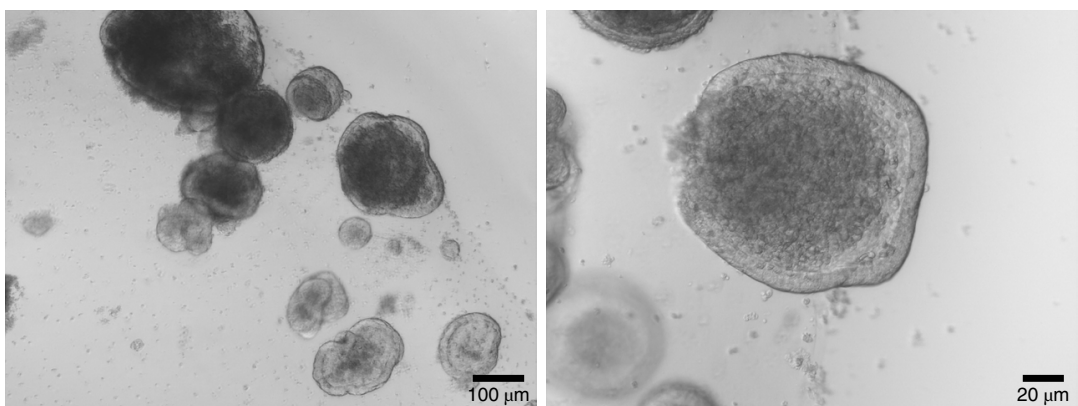

b

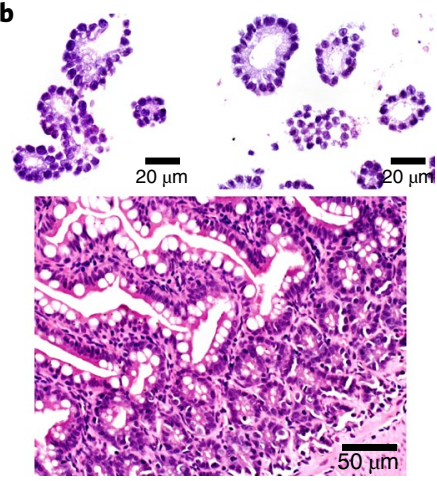

C
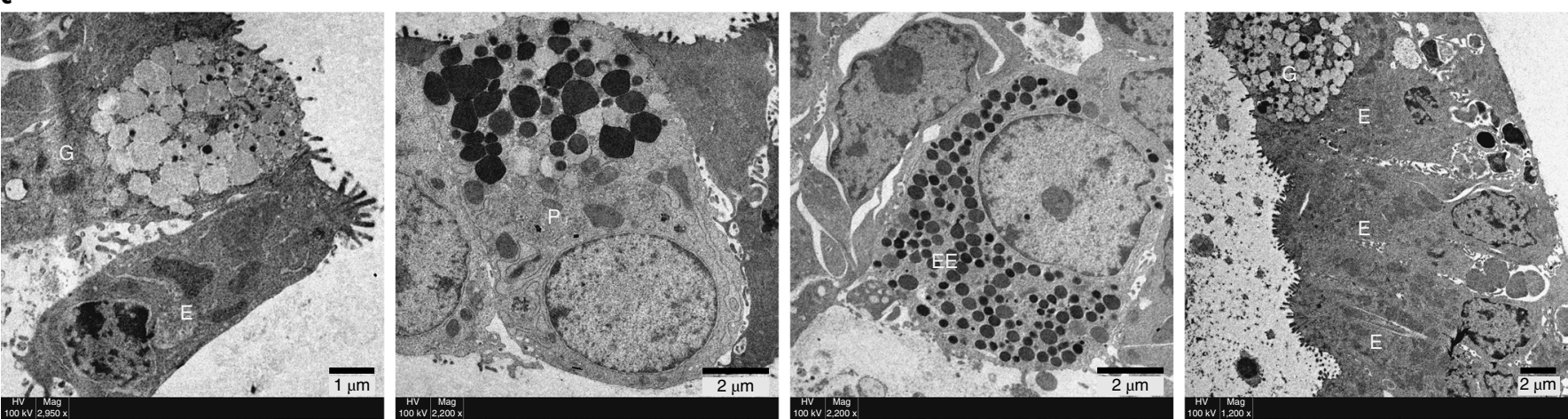

Fig. 1 | Morphological characterization of bat enteroids. a, Photomicrographs of differentiated bat enteroids. b, Photomicrographs of hematoxylin and eosin staining of bat enteroids (top) and bat small intestinal epithelium (bottom). c, Images of transmission electron microscopy illustrating ultrastructural morphology of absorptive Es, P cells, G cells and EE cells in differentiated bat enteroids. The photographs and images are representative of at least five similar images.

As shown in Fig. 1a, we successfully generated bat small intestinal organoids (enteroids) using the protocol for establishing human intestinal organoids ${ }^{20}$. The undifferentiated bat enteroids are propagated in expansion medium and passaged at a ratio of 1:2 every $7 \mathrm{~d}$. To induce differentiation, the expansion medium was replaced with differentiation medium, in which the enteroids were incubated for $4 \mathrm{~d}$. The differentiated bat enteroids mimic the multicellular composition of native bat small intestinal epithelium (Fig. 1b). Using transmission electron microscopy, we identified cells with characteristic features of four major intestinal cell types in the differentiated bat enteroids, including enterocytes (E), goblet $(G)$ cells, Paneth (P) cells and enteroendocrine (EE) cells (Fig. 1c). Although one line of bat enteroids was consecutively expanded for 12 weeks, the other lines ceased active proliferation after passage for 4 or 5 weeks, unlike human intestinal organoids, which can be consecutively passaged for at least 1 year. Nonetheless, we established the first bat intestinal organoid that simulates the cellular composition of bat intestinal epithelium.

We then assessed whether these bat enteroids are susceptible to SARS-CoV-2 and can be used for virus isolation. Enteric involvement has been previously reported in SARS-CoV and Middle East respiratory syndrome coronavirus (MERS-CoV) infections ${ }^{24,25}$. Accumulating evidence has suggested that some patients with COVID-19 have enteric infections ${ }^{4}$. Hence, we attempted virus isolation in differentiated bat and human enteroids simultaneously using clinical specimens, including nasopharyngeal aspirate (NPA) or sputum of three patients with COVID-19 from Queen Mary Hospital and Princess Margaret Hospital in Hong Kong (Extended Data Fig. 1). Both bat and human enteroids developed progressive cytopathic effect (CPE) after inoculation. Notably, a substantially increased viral load was observed in the culture media of bat and human enteroids over time (Fig. 2a). For example, inoculation of an NPA specimen in human enteroids resulted in a notably increased viral load of around $4 \log$ units in the culture media at $64 \mathrm{~h}$ after inoculation (hpi, Hu-ERD in Fig. 2a), whereas Vero E6 cells inoculated with the same specimen did not show $\mathrm{CPE}$ or increased viral load (data not shown). The culture media collected from the last timepoints (40 and $64 \mathrm{hpi}$ ) of the infected bat and human enteroids were used to inoculate a second batch of differentiated human enteroids. The medium collected from the infected bat organoids was subsequently inoculated into human organoids, not bat organoids, because we intended to inoculate the fresh media into the earliest available organoids to maximize the chance of virus isolation. A substantially increased viral load of more than $3 \log$ units in the culture media was observed after inoculation of the media from the first round of isolation (Fig. 2b). Immunofluorescence staining verified the presence of viral nucleocapsid protein (NP)-positive cells in human enteroids at the second round of inoculation (Fig. 2c).

We examined the human and bat enteroids by RT-PCR using a pair of degenerative primers targeting a highly conserved segment in several genera of coronaviruse ${ }^{26}$ and excluded the possibility that these organoids themselves carried any coronavirus (Supplementary Data Fig. 1). We did not compare the efficiency of virus isolation pairwise in Vero E6 cells and organoids using additional clinical specimens. However, based on our aforementioned observation (Hu-ERD in Fig. 2a and absence of CPE and viral growth in Vero E6 cells) and the fact that we used the patients' NPA or sputa instead of bronchioalveolar lavage fluid (specimen from the lower respiratory tract of a patient with severe disease $)^{1,2}$ for virus isolation, we think that both bat and human enteroids might enable virus isolation with higher efficiency than Vero E6 cells, which is the cell line commonly used for virus isolation. Zhu et al. also reported more efficient isolation of SARS-CoV-2 using human primary airway epithelial cells 

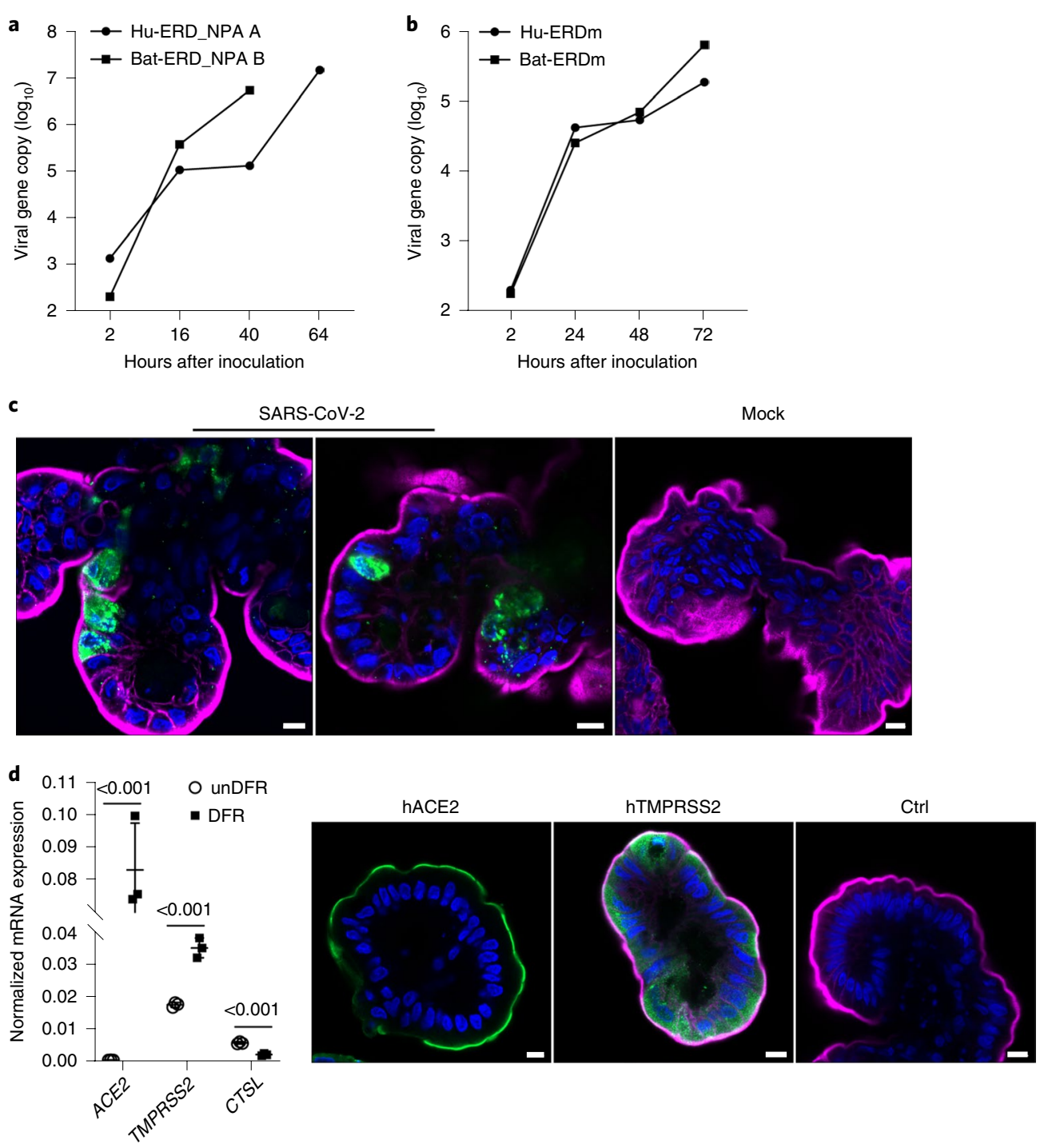

Fig. 2 | Highly effective virus isolation via bat and human enteroids. a, NPA specimens of two patients with COVID-19 were used to inoculate one droplet of bat enteroids (Bat-ERD_NPA B) or one droplet of human enteroids (Hu-ERD_NPA A). Cell-free media $(n=1)$ were harvested at the indicated hours after inoculation and followed by detection of viral gene copy. The data show the results of one specimen. $\mathbf{b}$, The media of the last timepoints of inoculated bat enteroids (Bat-ERDm) or human enteroids (Hu-ERDm) were used for the second round of inoculation in one droplet of human enteroids. Viral gene copies were detected in cell-free media $(n=1)$. c , The human enteroids in the second round of inoculation and mock-inoculated enteroids were fixed and subjected to immunostaining of viral NP (green). Nuclei and actin filaments were counterstained with DAPI (blue) and Phalloidin-647 (purple), respectively. Scale bar, $10 \mu \mathrm{m}$. d, Undifferentiated (unDFR) human enteroids and differentiated (DRF) enteroids of the same line were subjected to RT-qPCR to detect the transcriptional level of ACE2, TMPRSS2 and CTSL. Data represent the mean and s.d. of a representative experiment, $n=3$. Independent experiments were performed three times. Two-tailed Student's $t$-test. The differentiated human enteroids were subjected to immunofluorescence staining to label human ACE2 and TMPRSS2 protein (green). To explicitly show ACE2 expressed on cell surface membrane, the channel of actin is not incorporated into the image. The organoids stained with the second antibody only are imaged as a control (Ctrl). Scale bar, $10 \mu \mathrm{m}$. Images are representatives of at least five similar images.

than Vero E6 and Huh-7 cells ${ }^{2}$. Notably, our results indicate that both bat and human enteroids are susceptible to SARS-CoV-2.

SARS-CoV-2 uses the same receptor as SARS-CoV, human angiotensin-converting enzyme II (ACE2), for cell entry ${ }^{1}$. Apart from ACE2, transmembrane cellular protease human TMPRSS2 is required for priming SARS-CoV-2 for cellular entry ${ }^{27}$. In addition, human endosomal protease cathepsin L (CTSL) is involved in the entry of several coronaviruses, including SARS-CoV ${ }^{28}$ and MERS-CoV ${ }^{29}$. Given that the differentiated human enteroids sustain productive SARS-CoV-2 infection, we analyzed the expression of these entry factors. As shown in Fig. 2d, compared to undifferentiated enteroids, differentiated enteroids exhibited dramatically elevated ACE2 mRNA expression. The expression of TMPRSS2 was also significantly upregulated upon induction of differentiation, whereas that of CTSL was slightly downregulated. Figure $2 \mathrm{~d}$ shows the abundance of mRNA transcribed from these genes relative to that of highly expressed GAPDH. Thus, the differentiated human enteroids, which are used in all infection experiments, harbor abundant ACE2 and TMPRSS2 mRNA transcripts. Immunofluorescence staining also revealed abundant ACE2 and TMPRSS2 protein in the cells of differentiated human enteroids, consistent with their high expression in the human intestinal epithelium ${ }^{30}$. 

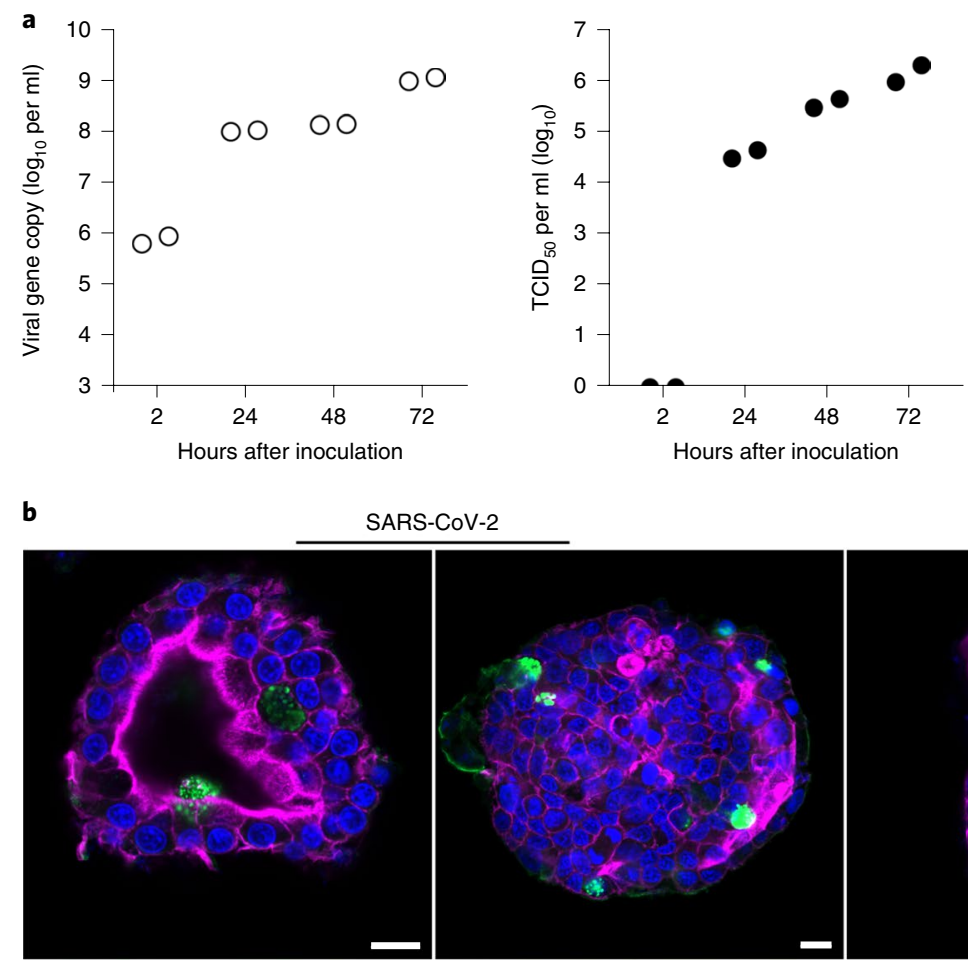

SARS-CoV-2

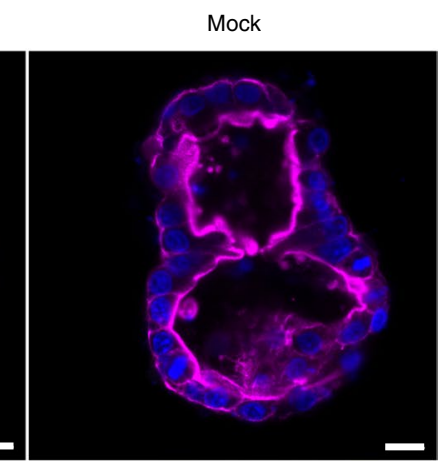

c
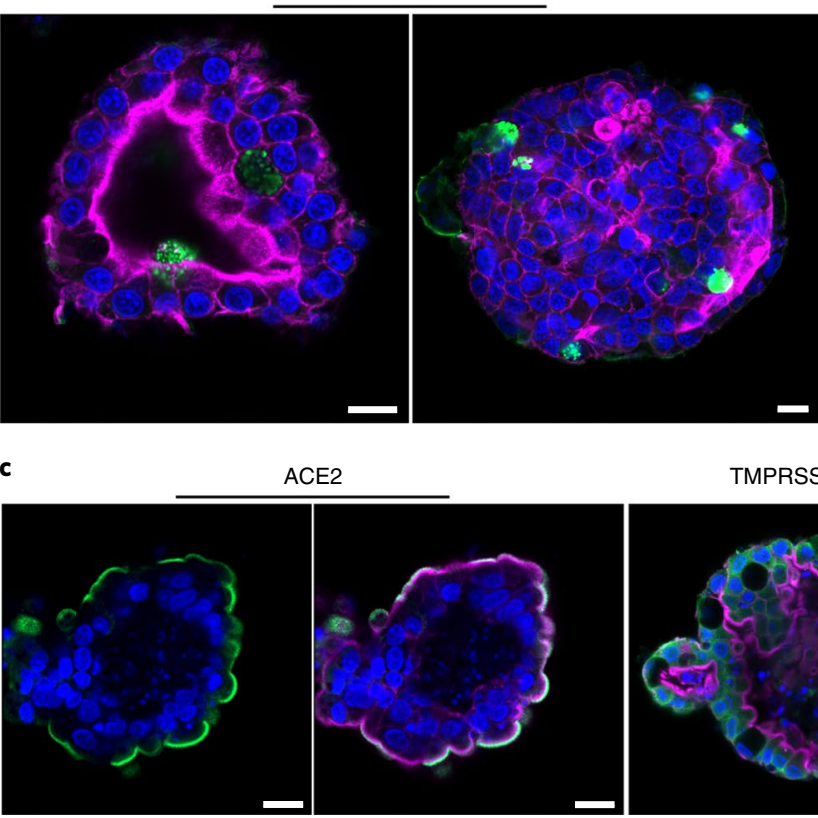

TMPRSS2

Ctrl

Fig. 3 | Active SARS-CoV-2 replication in bat enteroids. a, Culture media were harvested from the infected bat enteroids and subjected to viral load detection and viral titration by $T_{C I} D_{50}$ assay. Data represent the results of a representative experiment, $n=2$. Independent experiments were performed three times in duplicate or triplicate. b, SARS-CoV-2-infected or mock-infected bat enteroids were fixed and immunostained to identify viral NP (green)-positive cells. Nuclei and actin filaments were counterstained with DAPI (blue) and Phalloidin-647 (purple), respectively. c, Differentiated bat enteroids were fixed and immunostained using antibodies against human homologues to show ACE2 (green) and TMPRSS2 (green) protein. ACE2 is shown alone (left) and merged with actin (right). The organoids stained with the second antibody only are imaged as a control (Ctrl). Scale bar, $10 \mu \mathrm{m}$. Images are representatives of at least five similar images.

We then examined the replication kinetics of SARS-CoV-2 in differentiated bat enteroids. The infected bat enteroids gradually developed CPE at 72 hpi after inoculation with a multiplicity of infection (MOI) of 0.1 (results not shown). A notably increased viral load of more than $3 \log$ units was observed in the bat enteroids at $72 \mathrm{hpi}$ (Fig. $3 a)$. A $50 \%$ tissue culture infectious dose $\left(\mathrm{TCID}_{50}\right)$ assay conducted in Vero cells showed that the amounts of infectious virion particles substantially increased over time. Immunofluorescence staining for viral protein verified productive SARS-CoV-2 infection in the bat organoids. Viral NP-positive cells were discernible in infected enteroids but absent in mock-infected enteroids (Fig. 3b). We did not perform additional infection experiments using the supernatant of infected bat enteroids. Collectively, the results demonstrate that bat enteroids indeed sustain replication of SARS-CoV-2.

The whole genome of $R$. sinicus bat has been sequenced ${ }^{31}$ but is not yet annotated. We are therefore unable to assess mRNA expression of the entry factors in bat organoids owing to a lack of information on $R$. sinicus sequences for specific genes. Given the susceptibility of differentiated bat enteroids to SARS-CoV-2, we speculate that bat organoids might possess ACE2 and TMPRSS2 proteins similar to the human homologues that might be recognized by antibodies against human ACE2 and TMPRSS2. As shown in Fig. $3 c$, immunofluorescence staining showed ACE2 and TMPRSS2 in bat enteroids, with a similar distribution pattern to that of human enteroids (Fig. 2d). Sequence information of the least horseshoe bat (Rhinolophus pusillus) ACE2 is available in GenBank. Pairwise comparison between human and least horseshoe bat was performed using CLC Genomics Workbench 12.0.2 based on the mRNA and protein sequences of ACE2. ACE2 mRNA sequence of the least horseshoe bat (GenBank GQ999938.1) showed 79.5\% identity to the human homolog (AB046569.1), whereas the amino acid similarity was $80.5 \%$ between human (BAB40370.1) and least horseshoe bat (ADN93477.1).

Many animal coronaviruses primarily infect the enteric and respiratory tracts ${ }^{32,33}$. Enteric involvement has been reported in patients with SARS ${ }^{24}$. We previously demonstrated MERS-CoV replication in human intestinal organoids, and the human intestinal tract serves as an alternative route of MERS-CoV infection ${ }^{25}$. Given the effective 
a

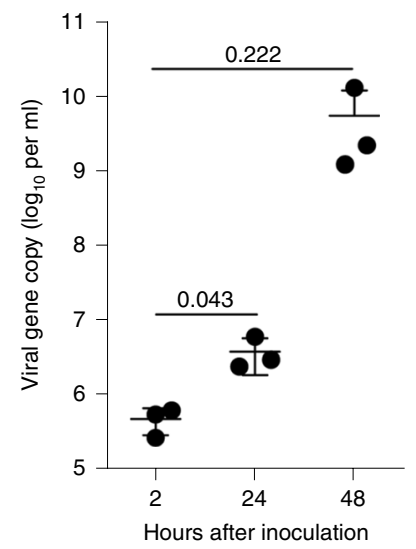

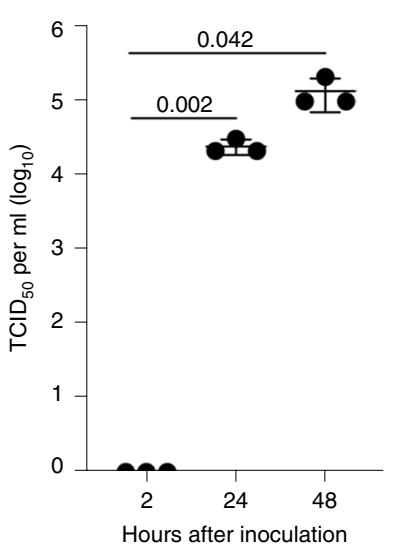

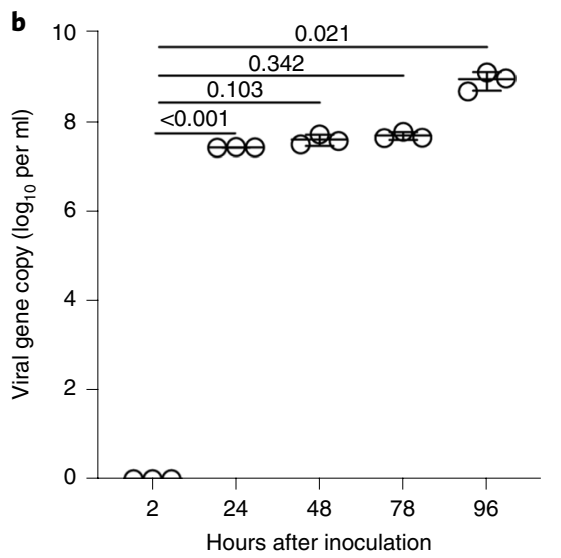

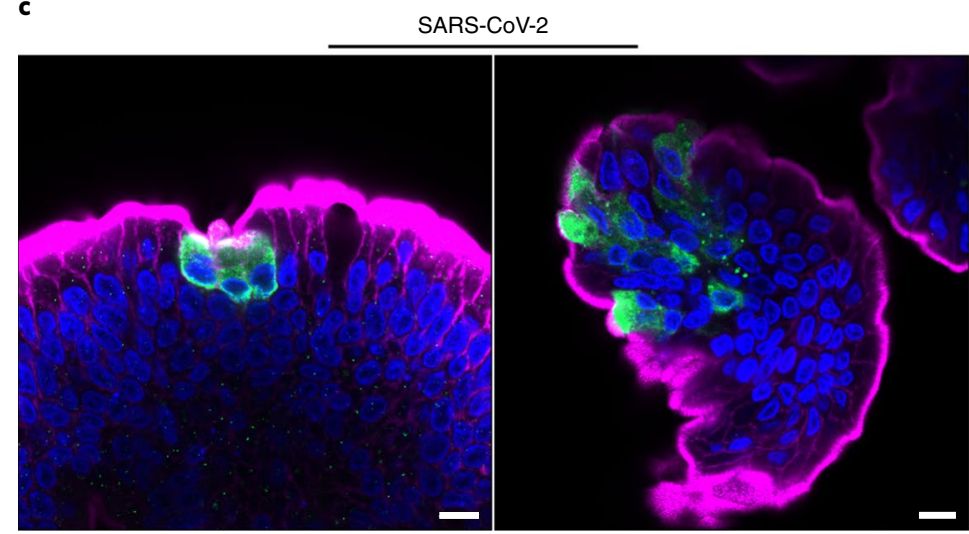

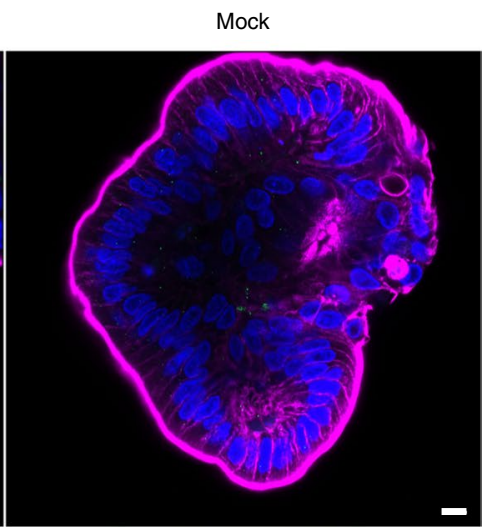

d

SARS-CoV-2

Mock

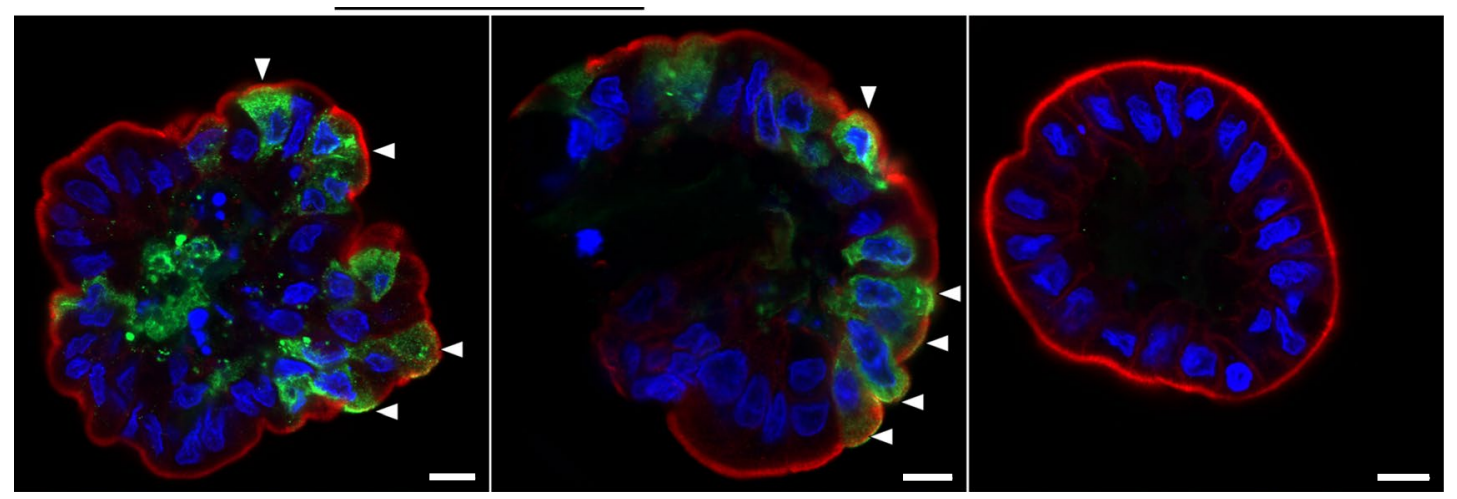

e

O Mock

Visualization of $\log _{2}$ (fold change)
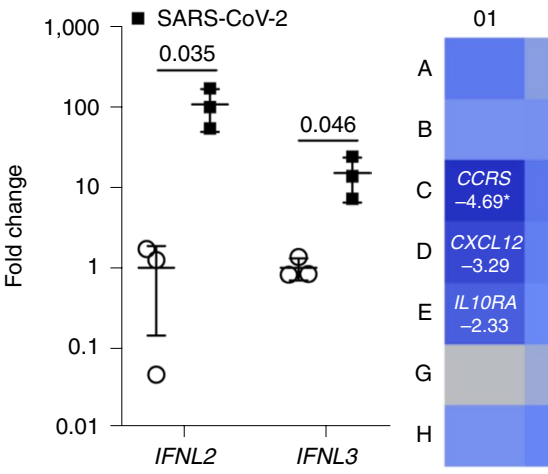

$05 \quad 06 \quad 07$
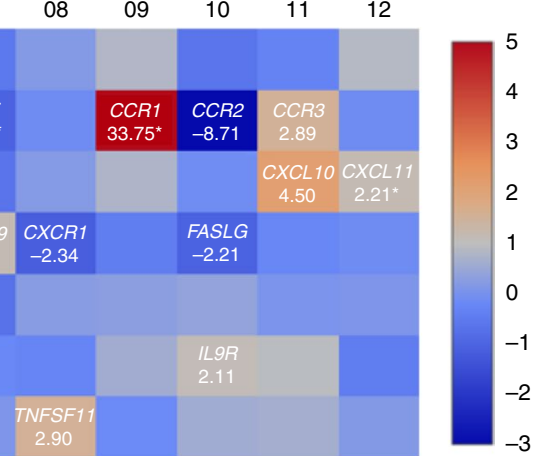

isolation of SARS-CoV-2 using differentiated human enteroids, we assessed viral replication kinetics. CPE developed sooner after inoculation in human enteroids than in bat enteroids. A significantly increased viral load of over $3 \log$ units was observed in human enteroids within $48 \mathrm{~h}$ after inoculation (Fig. 4a). Accordingly, virus titer significantly increased, as shown by $\mathrm{TCID}_{50}$ assay. We also evaluated virus growth 
Fig. 4 | Productive infection and cellular tropism of SARS-CoV-2 in human intestinal organoids. a, Culture media were collected from the infected human enteroids and subjected to viral load detection and viral titration. Data present the mean and s.d. of one representative experiment, $n=3$ replicates. Independent experiments were performed more than three times. Two-tailed Student's $t$-test. $\mathbf{b}$, Culture media collected from the inoculated colonoids were subjected to viral load detection. Data present the mean and s.d. of one representative experiment, $n=3$ replicates. Independent experiments were performed two times. c, The human enteroids were fixed after a low MOI (left) or high MOI (right) inoculation and subjected to immunostaining to identify the viral NP (green)-positive cells. Nuclei and actin filaments were counterstained with DAPI (blue) and Phalloidin-647 (purple), respectively. Scale bar, $10 \mu \mathrm{m}$. Independent experiments were performed more than three times. d, After a high MOI inoculation, the human enteroids were co-labeled with $\alpha-N P$ (green) and $\alpha$-Villin (red). Arrows show NP-positive cells coexpressing Villin. Scale bar, $10 \mu$ m. e, Induction of IFNL2 and IFNL3 in the human enteroids at 48 hpi. Results show fold change of GAPDH-normalized expression level in the infected enteroids relative to that in the mock-infected enteroids. Data represent mean and s.d. of one representative experiment, $n=3$ replicates. Independent experiments were performed three times. Two-tailed Student's t-test. The same RNA samples were applied to detect 84 human inflammatory cytokines and receptors. The heat map shows the differentially expressed genes in the infected enteroids $(n=3)$ relative to mock-infected organoids $(n=3)$. Genes with a fold change of more than 2 are illustrated; star $\left(^{\star}\right)$ indicates a change of significant difference.

in differentiated human colonoids, which are derived from crypts of human large intestines, by detecting viral load in the culture media. CPE appeared at $96 \mathrm{hpi}$ in differentiated human colonoids (results not shown). The human colonoids recapitulated robust viral replication with higher magnitude than in enteroids (Fig. 4b). Productive infection was also observed in human enteroids by immunofluorescence staining (Fig. 4c).

We proceeded to evaluate cellular tropism of SARS-CoV-2 in human enteroids. At $24 \mathrm{~h}$ after inoculation with an MOI of 3, we performed confocal imaging of the infected human enteroids after co-staining of viral NP and Villin, a marker of human enterocytes. As shown in Fig. 4d, most infected cells in the enteroids were Villin positive, indicating that enterocytes are the major target cell of SARS-CoV-2. This is conceivable because enterocytes are the dominant cell population in both differentiated human intestinal organoids and the native human intestinal epithelium. Type III IFNs have been shown to be selectively upregulated upon viral infections in human enteroids and human intestinal mucosa ${ }^{34,35}$. We then assessed type IIII IFN response in human enteroids upon SARS-CoV-2 infection. We also examined the expression of inflammatory cytokines and receptors in SARS-CoV-2-infected human enteroids in comparison with mock-infected enteroids using a quantitative multiplex assay: $\mathrm{RT}^{2}$ Profiler PCR Array Gene Expression Analysis. We found that the expression levels of genes encoding human IFN- $\alpha$, IFN- $\beta$ and IFN- $\gamma$ were barely induced upon infection (data not shown). Notably, human IFNL2 and IFNL3 were highly induced in the infected enteroids compared to controls of mock infection (Fig. 4e). Among the 84 interrogated genes in the PCR array, CCR1, CCR8, IL16, IL3 and CXCL10 (IP10) were upregulated, whereas CCR2, CCR5 and IL5 were downregulated. Further studies are required to elucidate the effect of regulation of these antiviral and inflammatory mediators on viral replication and host response.

Lastly, to investigate whether enteric infection of SARS-CoV-2 might occur in humans, we attempted virus isolation from the stool specimen of a patient with diarrheal COVID-19. A 68-year-old female patient presented with fever, sore throat and productive cough and developed diarrhea after admission to Princess Margaret Hospital. RT-qPCR of her stool specimen was positive for SARS-CoV-2, with a Ct value of 33.6. We isolated infectious virus from her stool specimen (Extended Data Fig. 1). The isolation of infectious virus from the patient's stool suggests that SARS-CoV-2 enteric infection occurred.

To summarize, we established the first organoid culture of bat intestinal epithelium. The differentiated bat enteroids recapitulate multicellular composition of bat intestinal epithelium and thus might serve as a robust in vitro model for studying microbial infections in bat intestine. Previous studies demonstrated that horseshoe bats are natural reservoirs of SARSr-CoVs ${ }^{12,13}$. A bat origin of SARS-CoV-2 has been proposed based on its high sequence homology to SARSr-CoVs identified in horseshoe bats ${ }^{1}$. The robust
SARS-CoV-2 replication in the enteroids derived from horseshoe bats suggests that bat intestinal cells might support natural infection by SARS-CoV-2 or its progenitor(s). Notably, these bat enteroids open up a new avenue for isolating and cultivating other bat viruses, which has been a longstanding challenge for studying numerous bat viruses of high zoonotic potential. However, the current medium cocktail to maintain bat enteroids is unable to consistently sustain long-term expansion as it does in human enteroids, suggesting the requirement for further optimization. In addition, the functionality of bat enteroids needs more characterization.

Although not as common as respiratory symptoms, gastrointestinal symptoms have been present in a substantial proportion of patients with COVID-19. Xiao et al. reported that $53 \%$ of a cohort of 73 hospitalized patients with COVID-19 had SARS-CoV-2 RNA in stool specimens, and stool remained positive in $23 \%$ of patients even after respiratory specimens became viral RNA negative ${ }^{3}$. Notably, viral NP-positive cells were observed in gastrointestinal epithelial cells from the biopsy specimens of these patients ${ }^{3}$. Persistent fecal viral shedding was also prominent in pediatric patients ${ }^{36}$. These clinical observations collectively suggest that enteric infection can occur in patients with COVID-19. Here we provide direct evidence of active SARS-CoV-2 replication in human enteroids, an in vitro model of human intestinal epithelium. The isolation of infectious virus from a patient with diarrheal COVID-19, coupled with these clinical observations, suggests that productive SARS-CoV-2 infection in human enteroids might recapitulate enteric infection in patients with COVID-19. The active viral replication and induction of type III IFNs and inflammatory mediators in human enteroids might contribute to the development of gastrointestinal symptoms in patients and requires further study. Currently, the route(s) leading to enteric infection remains unclear, such as whether the intestinal epithelial cells are primarily infected with SARS-CoV-2 via the oral-fecal route or whether enteric infection is secondary to respiratory infection. Nonetheless, we propose that the human intestinal tract might represent an additional route of virus transmission.

\section{Online content}

Any methods, additional references, Nature Research reporting summaries, source data, extended data, supplementary information, acknowledgements, peer review information; details of author contributions and competing interests; and statements of data and code availability are available at https://doi.org/10.1038/s41591 020-0912-6.

Received: 6 March 2020; Accepted: 24 April 2020; Published online: 13 May 2020

\section{References}

1. Zhou, P. et al. A pneumonia outbreak associated with a new coronavirus of probable bat origin. Nature 579, 270-273 (2020). 
2. Zhu, N. et al. A novel coronavirus from patients with pneumonia in China, 2019. N. Engl. J. Med. 382, 727-733 (2020).

3. Fei Xiao, Tang M., Zheng X., Liu Ye, Li X., Hong S. Evidence for gastrointestinal infection of SARS-CoV-2. Gastroenterology 158, 1831-1833 (2020).

4. Wang, D. et al. Clinical characteristics of 138 hospitalized patients with 2019 novel coronavirus-infected pneumonia in Wuhan, China. JAMA 323, 1061-1069 (2020).

5. World Health Organization. WHO COVID-19 Situation Report - 51 . https://www.who.int/docs/default-source/coronaviruse/situation-reports/20200 311-sitrep-51-covid-19.pdf?sfvrsn=1ba62e57_10 (2020)

6. Huang, C. et al. Clinical features of patients infected with 2019 novel coronavirus in Wuhan, China. Lancet 395, 497-506 (2020).

7. Rothe, C. et al. Transmission of 2019-nCoV infection from an asymptomatic contact in Germany. N. Engl. J. Med. 382, 970-971 (2020).

8. Chan, J. F. et al. Genomic characterization of the 2019 nove human-pathogenic coronavirus isolated from a patient with atypical pneumonia after visiting Wuhan. Emerg. Microbes Infect. 9, 221-236 (2020).

9. Lu, R. et al. Genomic characterisation and epidemiology of 2019 novel coronavirus: implications for virus origins and receptor binding. Lancet 395 , 565-574 (2020).

10. Zhou, P. et al. Fatal swine acute diarrhoea syndrome caused by an HKU2-related coronavirus of bat origin. Nature 556, 255-258 (2018).

11. Munster, V. J. et al. Replication and shedding of MERS-CoV in Jamaican fruit bats (Artibeus jamaicensis). Sci. Rep. 6, 21878 (2016).

12. Lau, S. K. et al. Severe acute respiratory syndrome coronavirus-like virus in Chinese horseshoe bats. Proc. Natl Acad. Sci. USA 102, 14040-14045 (2005).

13. Ge, X. Y. et al. Isolation and characterization of a bat SARS-like coronavirus that uses the ACE2 receptor. Nature 503, 535-538 (2013).

14. $\mathrm{Hu}, \mathrm{B}$. et al. Discovery of a rich gene pool of bat SARS-related coronaviruses provides new insights into the origin of SARS coronavirus. PLoS Pathog. 13 , e1006698 (2017)

15. Menachery, V. D. et al. A SARS-like cluster of circulating bat coronaviruses shows potential for human emergence. Nat. Med. 21, 1508-1513 (2015).

16. Banerjee, A., Misra, V., Schountz, T. \& Baker, M. L. Tools to study pathogen-host interactions in bats. Virus Res. 248, 5-12 (2018).

17. Yang, X. L. et al. Isolation and characterization of a novel bat coronavirus closely related to the direct progenitor of severe acute respiratory syndrome coronavirus. J. Virol. 90, 3253-3256 (2015).

18. Lau, S. K. et al. Ecoepidemiology and complete genome comparison of different strains of severe acute respiratory syndrome-related Rhinolophus bat coronavirus in China reveal bats as a reservoir for acute, self-limiting infection that allows recombination events. J. Virol. 84, 2808-2819 (2010).

19. Clevers, H. Modeling development and disease with organoids. Cell $\mathbf{1 6 5}$ 1586-1597 (2016)
20. Sato, T. et al. Long-term expansion of epithelial organoids from human colon, adenoma, adenocarcinoma, and Barrett's epithelium. Gastroenterology 141, 1762-1772 (2011)

21. Zhou, J. et al. Differentiated human airway organoids to assess infectivity of emerging influenza virus. Proc. Natl Acad. Sci. USA 115, 6822-6827 (2018).

22. Ettayebi, K. et al. Replication of human noroviruses in stem cell-derived human enteroids. Science 353, 1387-1393 (2016).

23. Heo, I. et al. Modelling Cryptosporidium infection in human small intestinal and lung organoids. Nat. Microbiol. 3, 814-823 (2018).

24. Leung, W. K. et al. Enteric involvement of severe acute respiratory syndrome-associated coronavirus infection. Gastroenterology 125, 1011-1017 (2003).

25. Zhou, J. et al. Human intestinal tract serves as an alternative infection route for middle east respiratory syndrome coronavirus. Sci. Adv. 3, eaao4966 (2017).

26. Woo, P. C. et al. Characterization and complete genome sequence of a novel coronavirus, coronavirus HKU1, from patients with pneumonia. J. Virol. 79, 884-895 (2005)

27. Hoffmann, M. et al. SARS-CoV-2 cell entry depends on ACE2 and TMPRSS2 and is blocked by a clinically proven protease inhibitor. Cell $\mathbf{1 8 1}$, 271-280 (2020).

28. Huang, I. C. et al. SARS coronavirus, but not human coronavirus NL63, utilizes cathepsin L to infect ACE2-expressing cells. J. Biol. Chem. 281, 3198-3203 (2006).

29. Park, J. E. et al. Proteolytic processing of middle east respiratory syndrome coronavirus spikes expands virus tropism. Proc. Natl Acad. Sci. USA 113 12262-12267 (2016)

30. Bertram, S. et al. Influenza and SARS-coronavirus activating proteases TMPRSS2 and HAT are expressed at multiple sites in human respiratory and gastrointestinal tracts. PLoS ONE 7, e35876 (2012).

31. Dong, D. et al. The genomes of two bat species with long constant frequency echolocation calls. Mol. Biol. Evol. 34, 20-34 (2017).

32. Openshaw, P. J. Crossing barriers: infections of the lung and the gut. Mucosal Immunol. 2, 100-102 (2009).

33. Saif, L. J. Bovine respiratory coronavirus. Vet. Clin. North Am. Food Anim. Pract. 26, 349-364 (2010).

34. Good, C., Wells, A. I. \& Coyne, C. B. Type III interferon signaling restricts enterovirus 71 infection of goblet cells. Sci. Adv. 5, eaau4255 (2019).

35. Wells, A. I. \& Coyne, C. B. Type III interferons in antiviral defenses at barrier surfaces. Trends Immunol. 39, 848-858 (2018).

36. $\mathrm{Xu}, \mathrm{Y}$. et al. Characteristics of pediatric SARS-CoV-2 infection and potential evidence for persistent fecal viral shedding. Nat. Med. 26, 502-505 (2020).

Publisher's note Springer Nature remains neutral with regard to jurisdictional claims in published maps and institutional affiliations.

(c) The Author(s), under exclusive licence to Springer Nature America, Inc. 2020 


\section{Methods}

Establishment, maintenance and differentiation of bat intestinal organoids. After ethics approval by the Agriculture, Fisheries and Conservation Department, Government of Hong Kong Special Administrative Region, three horseshoe bats (Rhinolophus sinicus) were captured and transferred to our laboratory. To establish bat intestinal organoids, we followed the same protocol for generating human intestinal organoids as described by Sato et al. ${ }^{20}$. In brief, the intestines of bats were harvested after the bats were euthanized by intraperitoneal injection of phenobarbital ( $200 \mathrm{mg} / \mathrm{kg}$ body weight). Intestinal tissues, after washing with cold PBS, were chopped into small pieces and incubated in cold chelation buffer $\left(5.6 \mathrm{mM} \mathrm{Na}_{2} \mathrm{HPO}_{4}, 8.0 \mathrm{mM} \mathrm{KH}_{2} \mathrm{PO}_{4}, 96.0 \mathrm{mM} \mathrm{NaCl}, 1.6 \mathrm{mM} \mathrm{KCl}, 44 \mathrm{mM}\right.$ sucrose and $54.8 \mathrm{mM} \mathrm{D}$-sorbitol in distilled water) for 30 minutes to isolate intestinal crypts. The isolated crypts were then pelleted by centrifugation at $200 \mathrm{~g}$ for 3 minutes, resuspended in cold Matrigel (Growth Factor Reduced Basement Membrane Matrix, Corning) and dispersed in a 24-well plate. After Matrigel was polymerized to form a droplet, $500 \mu \mathrm{l}$ expansion medium (shown in Table 1) was added to each well to maintain the culture at $37^{\circ} \mathrm{C}$ in a humidified incubator with $5 \% \mathrm{CO}_{2}$. To induce differentiation, the expansion medium was replaced with the differentiation medium (Table 1). Photomicrographs of the organoids were acquired using Nikon Eclipse TS100 Inverted Routine Microscope.

Maintenance and differentiation of human intestinal organoids. After ethics approval by the Institutional Review Board of the University of Hong Kong/ Hospital Authority Hong Kong West Cluster (UW13-364), human intestinal organoids were previously established using the small and large intestinal mucosa adjacent to the diseased tissues from pediatric patients who underwent surgical resection ${ }^{25}$. Two lines of human enteroid and one line of colonoid derived from three donors were used in this study. Detailed information of these donors is provided in Table 2. The expansion and differentiation of human intestinal organoids were performed with the same protocol as described above. Unless stated otherwise, all infections and examinations were conducted in the differentiated organoids of bat and human.

Table 1 | Composition of the expansion medium and differentiation medium for intestinal organoid culture

\begin{tabular}{|c|c|c|}
\hline Composition (concentration) & $\begin{array}{l}\text { Expansion } \\
\text { medium }\end{array}$ & $\begin{array}{l}\text { Differentiation } \\
\text { medium }\end{array}$ \\
\hline Advanced F12/DMEM & + & + \\
\hline $\begin{array}{l}\text { Wnt3a conditioned medium } \\
(50 \%)^{a}\end{array}$ & + & - \\
\hline $\begin{array}{l}\text { Rspondin1 conditional medium } \\
(20 \%)^{\mathrm{a}}\end{array}$ & + & - \\
\hline Noggin conditional medium (10\%) & + & - \\
\hline B27 supplement (1X) & + & + \\
\hline Nicotinamide (10 mM) & + & - \\
\hline A8301 (500 nM) & + & + \\
\hline SB202190 $(10 \mu M)$ & + & - \\
\hline hEGF (50 ng ml-1) & + & + \\
\hline $\mathrm{Y}-27632(10 \mu \mathrm{M})$ & + & + \\
\hline hGastrin I (10 nM) & + & + \\
\hline $\mathrm{N}$-acetylcysteine (1 mM) & + & + \\
\hline HEPES (10 mM) & + & + \\
\hline GlutaMAX (2 mM) & + & + \\
\hline Primocin $(50 \mu g)$ & + & + \\
\hline Penicillin-streptomycin $\left(100 \mathrm{U} \mathrm{ml}^{-1}\right)$ & + & + \\
\hline
\end{tabular}

apercentage of total volume.

Table 2 I Information on patients and the donated tissue from which the organoids were derived

\begin{tabular}{lllll}
$\begin{array}{l}\text { Organoid } \\
\text { type }\end{array}$ & Gender & Age & Intestine segment & Derivation time \\
\hline Enteroid line 1 & Female & 28 months & Small intestine & July 2017 \\
$\begin{array}{l}\text { Enteroid } \\
\text { line 3 }\end{array}$ & Male & 3 months & Small intestine & August 2017 \\
Colonoid line 3 & Female & 25 months & Colon & August 2019
\end{tabular}

Virus isolation, infection and detection. After ethics approval by the institutional review board of the University of Hong Kong/Hospital Authority Hong Kong West Cluster (UW 13-372), four archived specimens from patients with COVID19-NPA (Ct values of 19.1 and 22.2), sputum (Ct value of 32.1) or feces (Ct value of 33.6) - were obtained. Informed consent was waived because archived specimens were used for the study. The differentiated intestinal organoids of bat and human were sheared mechanically and incubated with the clinical specimens for $2 \mathrm{~h}$ at $37^{\circ} \mathrm{C}$. The inoculated organoids were re-embedded into Matrigel and then maintained in the differentiation medium. To assess replication kinetics, SARS-CoV-2 was inoculated in bat and human organoids at an MOI of 0.1 . At the indicated hours after inoculation, cell-free culture media were harvested and applied to RNA extraction using the MiniBEST Viral RNA/DNA Extraction Kit (Takara) and detection of viral loads (viral gene copy numbers) by one-step RT-qPCR assay (QuantiNova Probe RT-PCR kit, Qiagen; Table 3), as well as viral titration by TCID $_{50}$ assay, as described previously ${ }^{37}$. Briefly, serial ten-time dilution of culture media was inoculated in Vero cell monolayer in sextuplet and cultured in penicillin-streptomycin-supplemented DMEM. The monolayers were observed for CPE for 2-3 days. Viral titer was calculated with the Reed-Munch endpoint method. One TCID $_{50}$ is interpreted as the amount of virus that causes CPE in $50 \%$ of inoculated wells.

The infected or mock-infected human enteroids harvested at $48 \mathrm{hpi}$, undifferentiated and differentiated human enteroids of the same donor, were applied to RNA extraction, followed by reverse transcription using oligo(dT). The resultant cDNAs were used to measure mRNA expression levels of IFN, ACE2, TMPRSS 2 and CTSL by qPCR assay (Table 4 ) or to detect 84 human inflammatory cytokines and receptors with $\mathrm{RT}^{2}$ Profiler PCR Array (Qiagen). All experiments with live viruses were conducted in biosafety level 3 laboratories after approval by the Faculty of Medicine, The University of Hong Kong.

Histology examination, immunofluorescence staining and transmission electron microscopy. Bat intestinal tissues and bat organoids were applied to conventional tissue processing and hematoxylin and eosin staining after fixation with $4 \%$ paraformaldehyde (PFA). The virus-inoculated or mock organoids, after fixation, were applied to immunofluorescence staining using in-house-made antibodies against SARS-CoV NP and the goat-anti-rabbit IgG Alexa Fluor 488

(A-11034, Invitrogen) or goat-anti-mouse IgG Alexa Fluor 488 (A-11001, Invitrogen) to identify SARS-CoV-2-infected cells. The NP antibodies are cross-reactive to SARS-CoV-2 NP owing to the $93 \%$ amino acid identity to SARS-CoV NP (validation result provided upon request). To assess cellular tropism, at $24 \mathrm{~h}$ after SARS-CoV-2 inoculation with an MOI of 3, the differentiated human enteroids were fixed with $4 \%$ PFA and co-labeled with a mouse NP antibody and $\alpha$-Villin (ab130751, Abcam) overnight at $4{ }^{\circ} \mathrm{C}$, followed by staining with secondary antibodies. Other antibodies used in the study included $\alpha$-ACE2 (AF933, R\&D Systems), $\alpha$-TMPRSS2 (PA5-14264, Invitrogen), goat anti-rabbit IgG Alexa Fluor 594 (A-11037, Invitrogen) and donkey

Table 3 | RT-PCR assay for detection of SARS-Co-2 viral load with QuantiNova Probe RT-PCR kit

Primer/probe sequence

F: 5'-CGCATACAGTCTTRCAGGCT-3'

R: 5'-GTGTGATGTTGAWATGACATGGTC-3'

Pb: 5' FAM-TTAAGATGTGGTGCTTGCATACGTAGAC -IABkFQ-3'

Components

$2 \times$ buffer

Water

RT mix

Forward primer $(10 \mu \mathrm{M})$

Reverse primer $(10 \mu \mathrm{M})$

Probe (10-M)

Subtotal

RNA

\section{Cycling profile}

\section{Temperature}

$45^{\circ} \mathrm{C}$

$95^{\circ} \mathrm{C}$

$95^{\circ} \mathrm{C}$

$55^{\circ} \mathrm{C}$

$40^{\circ} \mathrm{C}$
Volume $(\mu \mathrm{l})$

10

1.2

0.2

1.6

1.6

0.4

15

5

$\begin{array}{ll}\text { Time } & \text { Cycle } \\ 0 \mathrm{~min} & 1 \\ 5 \mathrm{~min} & 1 \\ 5 \mathrm{~s} & 45 \\ 30 \mathrm{~s} & 45 \\ 30 \mathrm{~s} & 1\end{array}$




\section{Table 4 | Primer sequence for detection of cellular gene expression by RT-qPCR}

\begin{tabular}{|c|c|}
\hline Gene & Sequence \\
\hline \multirow[t]{2}{*}{ IFNL2 } & 5'-TCCAGTCACGGTCAGCA-3' \\
\hline & 5'-CAGCCTCAGAGTGTTTCTTCT-3' \\
\hline \multirow[t]{2}{*}{ IFNL3 } & 5'-TAAGAGGGCCAAAGATGCCTT-3' \\
\hline & 5'-CTGGTCCAAGACATCCCCC-3' \\
\hline \multirow[t]{2}{*}{ ACE2 } & 5'-CATTGGAGCAAGTGTTGGATCTT-3' \\
\hline & 5'-GAGCTAATGCATGCCATTCTCA-3' \\
\hline \multirow[t]{2}{*}{ TMPRSS2 } & 5'-CTTTGAACTCAGGGTCACCA-3' \\
\hline & 5'-TAGTACTGAGCCGGATGCAC-3' \\
\hline \multirow[t]{2}{*}{ CTSL } & 5'-TGGTGGCCTAATGGATTATGC-3' \\
\hline & 5'-ССTTCTCCTGCTTAGGGATGT-3' \\
\hline
\end{tabular}

anti-goat IgG Alexa Fluor 488 (A-11055, Invitrogen). Nuclei and actin filaments were counterstained with DAPI (Thermo Fisher Scientific) and Phalloidin-647 (Sigma-Aldrich), respectively. The confocal images were acquired using a Carl Zeiss LSM 800 confocal microscope.

The differentiated bat enteroids were embedded in resin after sequential fixation in $2.5 \%$ glutaraldehyde and $1 \%$ osmium. The ultrathin sections were stained with uranyl acetate and examined under a Philips CM 100 transmission electron microscope. All images presented in this manuscript, including photomicrographs of histology and electron microscopy and confocal images, were selected from at least five similar pieces with the same settings.

Statistical analysis. Statistical analysis was conducted using GraphPad Prism 7.0. The two-tailed Student's $t$-test was used to determine statistical significance. Numbers of replicates are indicated in the figure legends.

Reporting Summary. Further information on research design is available in the Nature Research Reporting Summary linked to this article.

\section{Data availability}

The data supporting the findings of this study are available from the corresponding authors upon written request.

\section{References}

37. Zhou, J. et al. Active replication of middle east respiratory syndrome coronavirus and aberrant induction of inflammatory cytokines and chemokines in human macrophages: implications for pathogenesis. J. Infect. Dis. 209, 1331-1342 (2014).

\section{Acknowledgements}

We thank the Center of PanorOmic Sciences and Electron Microscope Unit, Li Ka Shing Faculty of Medicine, University of Hong Kong, for assistance in confocal imaging and electron microscopy. We also thank Z. Shi for constructive discussion and critical revision of the manuscript; C.T. Shek and C.P. Tong for providing the horseshoe bats; and S. Sridhar for English language editing. This work was partly supported by funding from the Health and Medical Research Fund (project no. 19180392) of the Food and Health Bureau of the Hong Kong Special Administrative Region (HKSAR) to J.Z.; the Theme-based Research Scheme (T11-707/15-R) of the Research Grants Council, HKSAR, to K.Y.Y.; the High Level Hospital-Summit Program in Guangdong, The University of Hong Kong-Shenzhen Hospital, to K.Y.Y.; and donations from the Shaw Foundation Hong Kong, May Tam Mak Mei Yin, Richard Yu and Carol Yu, Michael Seak-Kan Tong, the Respiratory Viral Research Foundation Limited, Hui Ming, Hui Hoy and the Chow Sin Lan Charity Fund Limited and the Chan Yin Chuen Memorial Charitable Foundation to K.Y.Y.

\section{Author contributions}

J.Z. and K.Y.Y. designed and supervised the study. C.L., X.L., M.C.C., X.Z., D.W., Y.W., H.Chu, K.-H.C. and C.C.-Y.Y. performed the experiments. A.L. and A.J.Z. conducted the histological staining. J.-P.C. prepared the NP antibodies. J.Z., C.L., X.L., M.C.C. and X.Z. analyzed the data. I.H.-Y.C., K.K.-Y.W, J.F.-W.C., K.K.W.T. and O.T.-Y. T. provided the human tissues and clinical specimens. J.Z., C.L., X.L., H.Chen and K.Y.Y. wrote the manuscript.

\section{Competing interests}

The authors declare no competing interests.

\section{Additional information}

Extended data is available for this paper at https://doi.org/10.1038/s41591-020-0912-6. Supplementary information is available for this paper at https://doi.org/10.1038/ s41591-020-0912-6.

Correspondence and requests for materials should be addressed to J.Z. or K.Y.Y. Peer review information Alison Farrell is the primary editor on this article and managed its editorial process and peer review in collaboration with the rest of the editorial team.

Reprints and permissions information is available at www.nature.com/reprints. 


\begin{tabular}{llll}
\hline Patient \& Specimen & Organoid type & Viral load increase & Data presentation \\
\hline patient A NPA & human enteroid & $4 \log$ units & Shown Fig. 2a \\
patient A NPA & bat enteroid & $2 \log$ units & Not shown \\
patient B NPA & human enteroid & $4 \log$ units & Not shown \\
patient B NPA & bat enteroid & $4 \log$ units & Shown in Fig. 2a \\
patient C sputum & human enteroid & $2 \log$ units & Not shown \\
patient C sputum & bat enteroid & $2 \log$ units & Not shown \\
patient D stool & human organoid & $3 \log$ units & Described in the text \\
\hline NPA, nasopharyngeal aspirate. & &
\end{tabular}

Extended Data Fig. 1 | Summary. A brief summary of clinical specimens and experiment results. 


\section{Reporting Summary}

Nature Research wishes to improve the reproducibility of the work that we publish. This form provides structure for consistency and transparency in reporting. For further information on Nature Research policies, see Authors \& Referees and the Editorial Policy Checklist.

\section{Statistics}

For all statistical analyses, confirm that the following items are present in the figure legend, table legend, main text, or Methods section.

n/a Confirmed

Х The exact sample size $(n)$ for each experimental group/condition, given as a discrete number and unit of measurement

$\square$ \ A statement on whether measurements were taken from distinct samples or whether the same sample was measured repeatedly

$\square$ The statistical test(s) used AND whether they are one- or two-sided

Only common tests should be described solely by name; describe more complex techniques in the Methods section.

$\searrow \square$ A description of all covariates tested

$\triangle \square$ A description of any assumptions or corrections, such as tests of normality and adjustment for multiple comparisons

$\triangle$ A full description of the statistical parameters including central tendency (e.g. means) or other basic estimates (e.g. regression coefficient)

$\triangle \square$ AND variation (e.g. standard deviation) or associated estimates of uncertainty (e.g. confidence intervals)

For null hypothesis testing, the test statistic (e.g. $F, t, r$ ) with confidence intervals, effect sizes, degrees of freedom and $P$ value noted Give $P$ values as exact values whenever suitable.

Х $\square$ For Bayesian analysis, information on the choice of priors and Markov chain Monte Carlo settings

Х $\square$ For hierarchical and complex designs, identification of the appropriate level for tests and full reporting of outcomes

$\triangle \square$ Estimates of effect sizes (e.g. Cohen's $d$, Pearson's $r$ ), indicating how they were calculated

Our web collection on statistics for biologists contains articles on many of the points above.

\section{Software and code}

Policy information about availability of computer code

Data collection

Data analysis no software is used.

GraphPad Prism 7.0

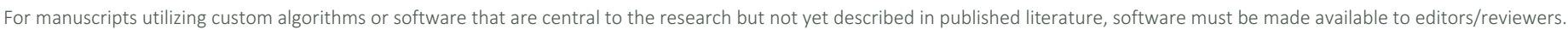
We strongly encourage code deposition in a community repository (e.g. GitHub). See the Nature Research guidelines for submitting code \& software for further information.

\section{Data}

Policy information about availability of data

All manuscripts must include a data availability statement. This statement should provide the following information, where applicable:

- Accession codes, unique identifiers, or web links for publicly available datasets

- A list of figures that have associated raw data

- A description of any restrictions on data availability

The data supporting the findings of this study are available from the corresponding authors upon written request.

\section{Field-specific reporting}

Please select the one below that is the best fit for your research. If you are not sure, read the appropriate sections before making your selection.

$\bigotimes$ Life sciences $\quad \square$ Behavioural \& social sciences $\quad \square$ Ecological, evolutionary \& environmental sciences

For a reference copy of the document with all sections, see nature.com/documents/nr-reporting-summary-flat.pdf 


\section{Life sciences study design}

All studies must disclose on these points even when the disclosure is negative.

Sample size No experiment presented in this study requires sample size to be determined

Data exclusions no excluded data

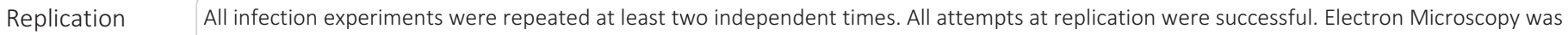
performed once, whereas there are at least 5 images for each cell type. The multiplex PCR array was performed one time in triplicate.

Randomization No experiment presented in this study requires randomization.

Blinding The results are either presented in quantitative data or with a negative control. Blinding is not required.

\section{Reporting for specific materials, systems and methods}

We require information from authors about some types of materials, experimental systems and methods used in many studies. Here, indicate whether each material, system or method listed is relevant to your study. If you are not sure if a list item applies to your research, read the appropriate section before selecting a response.

\begin{tabular}{|c|c|}
\hline$n / a$ & Involved in the study \\
\hline & $\bigotimes$ Antibodies \\
\hline & ¿ Eukaryotic cell lines \\
\hline$\bigotimes$ & Palaeontology \\
\hline & $\bigotimes$ Animals and other organisms \\
\hline L & $\bigotimes$ Human research participants \\
\hline Х & $\square$ Clinical data \\
\hline
\end{tabular}

Methods

$\mathrm{n} / \mathrm{a}$ Involved in the study

Х $\square$ ChIP-seq

Х $\square$ Flow cytometry

Х $\square$ MRI-based neuroimaging

\section{Antibodies}

Antibodies used

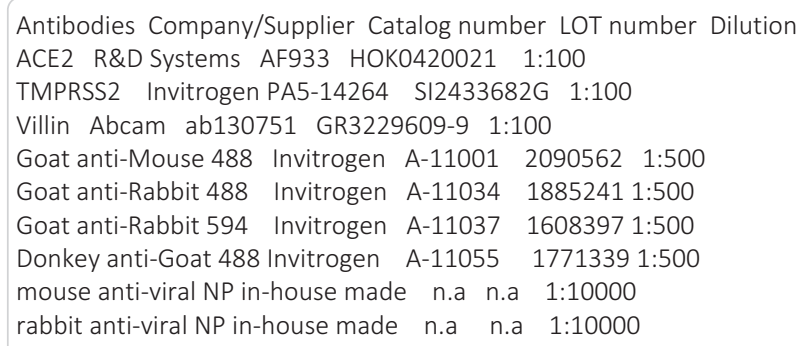

Validation

1) the application of commercial antibodies for IF staining has been validated by the providers

2) the validation result of in-house generated antibodies will be provided upon request.

\section{Eukaryotic cell lines}

Policy information about cell lines

Cell line source(s)

Vero cells from ATCC (https://www.atcc.org/products/all/CCL-81.aspx)

\section{Authentication}

Mycoplasma contamination

Commonly misidentified lines (See ICLAC register)
The cells are purchased from ATCC.

we verified the cells free of mycoplasma contamination by using the mycoplasma screening service provided by HKU core facilities.

No commonly misidentified lines were used. 
Policy information about studies involving animals; ARRIVE guidelines recommended for reporting animal research

Laboratory animals

Wild animals

Field-collected samples

Ethics oversight no lab animal involved.

Three female and male horseshoe bats (Rhinolophus sinicus) were captured in roosting caves with hand-nets on long pole by bat experts of Agriculture, Fisheries and Conservation Department, the Government of Hong Kong Special Administrative Region. The captured bats were kept in cloth bags individually and were transferred by the technical staff to HKU laboratory right after the capture. The organs were applied to establishing organoid culture after the bats were euthanized in biosafety hood by intraperitoneal injecting overdosed pentobarbitone sodium (100-150mg/kg). We have obtained the ethnic approval for this study.

\section{no field-collected sample involved}

Approved by Agriculture, Fisheries and Conservation Department, the Government of Hong Kong Special Administrative Region,

Note that full information on the approval of the study protocol must also be provided in the manuscript.

\section{Human research participants}

Policy information about studies involving human research participants

Population characteristics

Four COVID-19 patients who were confirmed by laboratory test. This study only used the patients' respiratory and fecal specimens for virus isolation and analysis. Patients themselves were not involved in any experiments.

Recruitment

Ethics oversight
The patients are confirmed cases of SARS-CoV-2 infection in two public hospital in Hong Kong.

Institutional Review Board of the University of Hong Kong/Hospital Authority Hong Kong West Cluster (UW 13-372)

Note that full information on the approval of the study protocol must also be provided in the manuscript. 